\title{
Robust Analysis and Control of Stochastic Interval Systems
}

\author{
Cheng Wang ${ }^{1,2}$, Congjun Rao ${ }^{1}$ \\ ${ }^{1}$ College of Mathematics and Computer Science, Huanggang Normal University, \\ Huanggang 438000, China \\ ${ }^{2}$ School of Automation, Huazhong University of Science and Technology, \\ Wuhan 430074, P. R. China \\ wangc80@163.com
}

\begin{abstract}
Keywords: Stochastic interval systems; Robust stability; Non-fragile control; Passive control Abstract. Stochastic interval systems is a new type of stochastic system in recent years, which is a new field integrated by information and control. This paper introduces the research history and the newest research trends of stochastic interval systems, and presents many widespread theoretical and application problems. Moreover, some new research topics and directions related to stochastic interval systems are proposed.
\end{abstract}

\section{Introduction}

For many problems in natural world, socio-economic, material systems of practical engineering, their dynamic rules can be described by deterministic dynamic model or random system model. The description of deterministic system model is by deterministic ordinary differential equations, which is to simplify the process of the actual system. Its system model is relatively simple, and is easy to analyze and synthesize, and its research tool is also relatively simple. When the considered system is under ideal conditions or system accuracy is not required, the system is often reduced to a deterministic model.

Since the creation of modern control theory, control theory based on deterministic system model has been developing rapidly, and played an important role in practical applications. However, in many practical systems, such as engineering controls, traffic management, physical circuit system, the random factor is objective and inevitable. Due to the perturbations by the internal structure parameter, the system control inputs and the external environment, the established model can not accurately describe the controlled object or the system creates deviation between the running model and the actual object. In large-scale integrated circuits, interference of random factors is inevitable. To ignore the interference of random factors will increase the systematic errors and affect system performance. On the radio circuit receiving system, due to the random noise disturbance outside, the received signal of a radio is unstable. Thus, random factors are worth considering.

With the development of science and technology, the system accuracy requirements of practical engineering technology become more and more high. The original simplified deterministic model can not meet the accuracy requirements of the system. The system designed by deterministic model in operation will deviate from predetermined design requirements, resulting in the generation of random deviation. Therefore, in the system modeling, we should fully consider the impact of random factors on the system, and use a stochastic model for system modeling and use the stochastic point of view for the system analysis and synthesis. Stochastic system model is described by stochastic ordinary differential equations. In a sense, the theory of stochastic systems are the combination product of deterministic systems theory and stochastic processes.

In most actual operation of the control system, there are more or less uncertain factors, such as the measurement error of physical components, the impact of limited word length of memory, and outside interference, and thus to establish accurate mathematical model of system objects is usually not possible. The uncertainty of system parameters causes a certain interference for the parameters of state-space model, which is an important reason to cause system instability and performance degradation. Parameter uncertainty is inevitable in the real system, where there is a kind of uncertainty 
can be described as a state of some or all of the elements of the matrix system changes in some defined interval, which is the interval control systems.

Interval control system is a kind of important systems with uncertain parameters. In a practical system, for example, the perturbation of system parameters induced by component aging, the changes of linear models for a aircraft caused by different heights and different Mach, which can be described by using interval system. For another example, the aircraft movement system and the motor control system can be regarded as the interval control system. In recent years, robust stabilization and interference suppression of interval systems became the focus of attention of many scholars, and made many valuable results [1-2], such as Ref. [3] pointed out that the supply chain system has the characteristics of uncertain stochastic systems with time-delay, and Ref. [4] studied stochastic stability problem for a supply chain system with the characteristics of interval gray time-delay, which shows that this kind of the interval control systems have important applications background.

\section{Robust Control and Stability Analysis of Stochastic Systems}

Random factors are widespread in nature. In 1931, Kolomogrov laid the foundation mathematical theory of stochastic processes. In 1951, Doob described the mathematical theory of stochastic processes. In 1951, It $\hat{o}$ first introduced the It $\hat{o}$ law, and correctly explained the meaning of stochastic differential [3], thus contributed to the great development of the theory of stochastic differential equations, and laid the theoretical foundation of stochastic differential equations. In 1969, Kozin [4] divided random system into two categories according to the distribution of the random variable, as well as the memory characteristics of random process, i.e., the stochastic systems which random parameter is Gaussian white noise, and the stochastic systems which random parameter is non-Gaussian white noise. For the stochastic systems which random parameter is Gaussian white noise, the mathematical model is It $\hat{o}$ stochastic differential equations.

Stochastic systems widely exist in the systems of natural science, engineering technology and society.

To study on the stochastic systems becomes very important research content for the engineering technology technicist. In recent years, some important research in the field of deterministic system has been successfully extended to stochastic systems. For example, [5-6] studied the stability for stochastic delay systems and random Markov delay systems by introducing free weighting matrices, and obtained corresponding delay-dependent stability criteria respectively. [7] discussed the stability of neutral stochastic uncertain delay systems. [8] extended the integral inequality method to the nonlinear stochastic uncertain delay systems, and obtained a delay-dependent stability criteria. [9] studied the robust exponential stability of a kind of neutral stochastic Markov jump systems, and gave the sufficient conditions of robust exponential stability for the systems. [10] studied the stability of a kind of neutral stochastic neural networks with Markov jump by using the segmentation method. Due to limitations of stochastic Lyapunov stability theory, the robust control of stochastic systems has been unable to get faster development. Until 1998, Hinrichsen [11] et al. put forward $\mathrm{H} \infty$ control of stochastic systems and established a random bounded real lemma, then the Hळ control of stochastic system has been greatly developed. With the development of $\mathrm{H} \infty$ control of stochastic systems, the research on stochastic systems has become a hotspot of research, and the stability and robust control problems of stochastic systems made great development, and a large number of research results emerged, for example, [12] studied robust $\mathrm{H} \infty$ control for the uncertain stochastic system with time-varying delay, and gave the sufficient conditions for the delay-independent and delay-dependent. [13] studied the robust stability and $\mathrm{H} \infty$ for the stochastic systems with time-varying delays. [14] studied the output feedback $\mathrm{H} \infty$ control for the stochastic delay systems and the state feedback stabilization state feedback $\mathrm{H} \infty$ control problems for the nonlinear stochastic systems.

In the above designing scheme of robust control, they only considered the uncertainty of system parameters, and did not consider the uncertainty of controller gain. Due to environmental changes, the system noise, hardware and software and other factors, there are uncertainties in the implementation process of controller, then the traditional method of robust control design exhibited a high degree of 
vulnerability, resulting in performance degradation or the stability of closed-loop system is destroyed, then the controller must be designed to withstand a certain degree of change. Therefore, the study of non-fragile controller received considerable attention, and a lot of achievements has presented, for example, [15] studied the Non-fragile robust Hocontrol problems for the stochastic delay systems, and gave the design method of non-fragile controller. [16] studied the non-fragile robust stabilization problem for the convex polyhedron uncertain stochastic systems, and gave non-fragile robust stabilization conditions.

Passivity was first introduced in the control theory by Lurie and Popov, which is the product of the input and output as an energy supply rates. Passivity is a special case of the dissipation. In 1972, Willems put forward the theory of dissipative systems, and established the relationship between a storage function and supply rate, and presented the concept of dissipation. It has a very close relationship between the passivity and stability of the system. For decades, through the efforts of scholars, the fruitful results are achieved, for example, [17] studied the passive control issues of the stochastic delay systems with time delay.[18] studied the passive analysis and control problems for the stochastic delay systems, and gave the passive conditions, and designed passive controller.

\section{Robust Control and Stability Analysis of Interval Systems}

In 1978, Kharitonov [19] proposed Four Endpoint Inspection Theorem on the robust stability of interval polynomials, which pioneered the study of interval systems. Kharitonov Theorem provided a kind of research ideas, i.e., sub-set test instead of the overall test, which regarded the interval system as a deterministic system. Inspired by Kharitonov's work, some researchers are trying to develop similar results for a kind of more sophisticated range of systems. For example, Bialas [20] studied the stability of the interval matrices which the elements changes in an interval. Hollot [21] studied the robust stability of polynomial polytopes and proposed the Edge Theorem.

As a basic tool for analyzing system stability, the traditional method of Lyapunov is natural to be applied to the robust stability analysis of interval systems. When using Lyapunov method, one idea is to create a Lyapunov function, and then estimate the stable area of the system in the parameter space. If the range of parameters is included in the estimated area, the system is robust and stable. Another idea is to create a Lyapunov function for the entire range of system, then to determine the robust stability of the system by testing whether the derivative is negative in the range of parameter variation. The benefits of using Lyapunov method is that it can analyze more general systems, but the other methods are difficult to effectively do this analysis. However, the criterion by the Lyapunov methods are generally sufficient, more or less have a certain conservative. To reduce conservatism, Barmish [22] proposed the concept of parameter dependent Lyapunov function, and used a parameter dependent Lyapunov function as a tool, and combined with LMI (Linear Matrix Inequality, LMI) method, then numerous sufficient robust stability criterion are obtained [23-24].

\section{New Research Topics and Directions}

As previously mentioned, there are series of results on the stochastic systems and the interval system, but the study of stochastic interval systems are rare. Because of issues related to stochastic interval systems research is more difficult, not many tools available. Therefore, the study of robustness and associated control problems for stochastic interval systems is necessary and urgent. So we can do this research work.

Firstly, we can use the LMI and generalized Itô formula to study the robust stability and robust stabilization problems for the, and establish the stochastic interval bounded real lemma for the, and then apply the lemma to the study of robust $\mathrm{H} \infty$ control and non-fragile $\mathrm{H} \infty$ control of linear stochastic interval systems, and design to state feedback controller which meets the performance criteria under the robust, stochastic dynamic feedback controller and a non-fragile controller. Secondly, we can study the passive control problems for the linear stochastic interval systems, establish the stochastic passive conditions, and design the randomized passive controller, and then extend the above results and the 
methods to the stochastic interval delay systems and neutral stochastic interval delay systems. For the research on nonlinear stochastic interval systems, we can comprehensively apply the stochastic analysis tools and modern control theory such as Itô formula, Finsler Lemma, Stochastic Integral Inequality and Random Razumikhin Theorem to establish the criterion for robust stability and stabilization of this kind of systems and bounded real Lemma, and study the robustness $\mathrm{H} \infty$ control, non-fragile $\mathrm{H} \infty$ robust control, passive control problem, and then design the appropriate controller and nonlinear controller. This study will enrich the random interval system robustness analysis and related control theory, and provides new ways and methods for in-depth study of stochastic interval control systems.

\section{Acknowledgments}

This work is supported by the National Natural Science Foundation of China (No. 61403153), the Natural Science Foundation of Hubei Province (No. 2015CFB420), China Postdoctoral Science Foundation (No. 2013M530344), and the Reform Project of Transformation Development of Huanggang Normal University (No. xfg2015C19).

\section{References}

[1] S. Hu and J. Wang, On stabilization of a new class of linear time-invariant interval systems via constant state feedback control, IEEE Transactions on Automatic Control 45 (2000) 2106-2211.

[2] W. Mao and Chu J. Quadratic stability and stabilization of dynamic interval systems. IEEE Transactions on Automatic Control, 2003, 48(6): 1007-1012.

[3] X.H. Ji, R.Q. Chen, and Z.J. Tang, The development and prospect of postponement research, Journal of Industrial Engineering 21 (2007) 62-66.

[4] X.L. Zhang, D.P. Wang, Z.Z. Zhao, Modeling and its stability analysis chain with grey and time delay properties, Industrial Engineering 15 (2012) 20-23.

[5] H.C. Yan, X.H. Huang, H. Zhang, M.Wang, Delay-dependent robust stability criteria of uncertain stochastic systems with time-varying delay, Chaos Solitons Fractals 40 (2009) 1668-1679.

[6] D. Yue, Q.L. Han, Delay-dependent exponential stability of stochastic systems with time-varying delay, nonlinearity, and Markovian switching, IEEE Transactions on Automatic Control 50 (2005) 217-222.

[7] F.Q. Deng, W.H. Mao, A.H. Wan, A novel result on stability analysis for uncertain neutral stochastic time-varying delay systems, Applied Mathematics and Computation 221 (2013) 132-143.

[8] Y. Chen, A.K. Xue, An improved stability criterion for uncertain stochastic delay systems with nonlinear uncertainties, IET Electronics Letters 44(2008) 458- 459.

[9] Y.J. Li, F.Q. Deng, The robust exponential stability for a class of uncertain neutral stochastic system with Markovian jump and time-varying delays, Journal of Systems Science and Complexity 32 (2012) 821-830.

[10] R. Rakkiyappan, Q.X. Zhu, A. Chandrasekar, Stability of stochastic neural networks of neutral type with Markovian jumping parameters: A delay-fractioning approach, Journal of Franklin Institute 351 (2014) 1553-1570.

[11] D. Hinrichsen, A.J. Pritchard, Stochastic Hळ. SIAM Journal of Control Optimization 36(1998) 1504-1538.

[12] S.Y. Xu, T.W. Chen, Robust Hœ control for uncertain stochastic systems with state delay, IEEE Transactions on Automatic Control 47(2002) 2089-2094. 
[13] J.T. Sun, Q.G. Wang, H.Q. Gao, Delay-dependent robust stability and $\mathrm{H} \infty$ analysis of stochastic systems with time-varying delay, Applied Mathematics and Mechanics 31(2010) 236-244.

[14] S.Y. Xu, T.W. Chen, Hळo output feedback control for uncertain stochastic systems with time-varying delays, Auotmatica 40(2004) 2091-2098.

[15] S.Y. Xu, L. Jam, G.H. Yang, J. Wang, Stabilization and Hळ control uncertain stochastic time-delay systems via non-fragile controllers, Asian Journal of Control 8 (2006) 197-200.

[16] W.H. Gao, F.Q. Deng, R.Q. Zhang, Non-fragile robust stabilization for stochastic delay-varying systems with polytopic-type uncertainties, Systems Engineering and Electronics 33(2011) 146-150.

[17] H.L. Liu, G.R. Duan, L.Y. Fan, Delay-dependent passivity control of stochastic differential system with time delay, Proceedings of the 8th World Congress on Intelligent Control and Automation 2010, pp. 963-968.

[18] Y. Chen, A.K. Xue, J.H. Wang, Delay-dependent passive control of stochastic systems with time-delay, Acta Automatica sinica 35(2009) 324-327.

[19] V.L. Kharitonov, Asymptotic stability of an equilibrium position of a family of systems of linear differential equations, Differentsial'nye Uravneniya 14 (1978) 2086-2088.

[20] S. Bialas, A necessary and sufficient condition for the stability of interval matrices, International Journal of Control 37 (1983) 717-722.

[21] A.C. Bartlett, C.V. Hollot, H. Lin, Root locations of an entire polytope of polynomials: it suffices to check the edges, Mathematics of Control, Signals, and Systems 1 (1988) 61-71.

[22] B.R. Barmish, C.L. DeMarco, A new method for improvement of robustness bounds for linear state equations, Proceedings of the 1986 Conference on Information Sciences and Systems. Princeton: Princeton University, 1986, pp. 115-120.

[23] R. Oliveira, P. Peres, Parameter-dependent LMIs in robust analysis: characterization of homogeneous polynomially parameter-dependent solutions via LMI relaxations, IEEE Transactions on Automatic Control 52(2007) 1334-1340.

[24] P. Seiler, U. Topcu, A. Packard, Parameter-dependent Lyapunov functions for linear systems with constant uncertainties, IEEE Transactions on Automatic Control 54 (2009) 2410-2416.

[25] X. Mao, J. Lam, S. Xu, H. Gao, Razumikhin method and exponential stability of hybrid stochastic delay interval systems, Journal of Mathematical Analysis and Applications 314 (2006) 45-66.

[26] H.N. Leng, Q. Zhang, An iterative algorithm for the stability analysis of dynamic interval systems, Applied Numerical Mathematics 69 (2013) 73-77.

[27] A.U. Udom, Exponential stabilization of stochastic interval system with time dependent parameters, European Journal of Operational Research 222 (2012) 523-528.

[28] C. Wang, Y. Shen, Passive control for stochastic interval systems with interval time-varying delay, Asian Journal of Control 15 (2013) 248-259. 